\title{
Analysis of the Symmetry Properties of Large Periodic Magnetic Systems, to reduce the Computation Time of the Calculation of the Magnetostatic Dipolar Energy
}

\author{
Iván Cabria \\ Departamento de Física Teórica, Atómica y Óptica, Universidad de Valladolid, 47011 Valladolid, Spain \\ Email: ivan.cabria@uva.es
}

\begin{abstract}
The computational effort to calculate the magnetostatic dipolar energy, MDE, of a periodic cell of $N$ magnetic moments is an $O\left(N^{2}\right)$ task. Compared with the calculation of the Exchange and Zeeman energy terms, this is the most computationally expensive part of the atomistic simulations of the magnetic properties of large periodic magnetic systems. To reduce the computational effort, the traditional Ewald method to calculate the MDE of periodic magnetic systems has been analyzed. The detailed analysis reveals that, for certain types of periodic systems, there are many matrix elements of the Ewald method identical to another elements, due to symmetry properties of the periodic systems. Computation timing experiments of the MDE of large systems, such as Ni fcc nanowires up to 31500 magnetic moments in the periodic cell, have been carried out and they show that the number of matrix elements that should be calculated is approximately equal to $N$, instead of $N^{2} / 2$, if these symmetries are used, and that the computation time decreases in an important amount. The time complexity of the analysis of the symmetries is $O\left(N^{3}\right)$, which increases the time complexity of the traditional Ewald method and is in contrast with the computation timing experiments. This is explained by the fact that the MDE is a very small energy and therefore, the usual required precision of the calculation of the MDE is so high, about $10^{-6} \mathrm{eV} /$ cell, that the calculations of large periodic magnetic systems are very expensive and the use of the symmetries reduces, in practical terms, the computation time of the MDE in a significant amount, in spite of the increase of the time complexity.
\end{abstract}

Index Terms-Magnetostatic dipolar energy, magnetostatic dipolar anisotropy energy, magnetic anisotropy energy, magnetic layered materials, nanowires, ferromagnetic materials, Ewald method

\section{Introduction and Motivation}

The most expensive part of the atomistic simulations of the magnetic properties of periodic magnetic systems of certain thickness, such as nanowires and films of ferromagnetic atoms, is the calculation of the magnetostatic dipolar energy, MDE [1], [2], [3], [4], [5], [6], [7]. To simulate these materials with realistic models, it is necessary to consider a large number of atoms and the details of the geometric structure. For instance, in the case of arrays of magnetic nanowires, it is important to consider the structure in the edges or surface of the nanowires and the distances between the walls of the nanowires in the array. However, calculations of the MDE of systems with a large number of atoms are very expensive.

An analysis of the Ewald method in its traditional form [8], [9], [10], [11] to calculate the MDE of periodic magnetic systems has been carried out, finding that many matrix elements are identical to others depending on the type of Bravais lattice cell and if the basis atoms of the cell of the periodic magnetic system satisfy certain conditions or symmetries. When these symmetries are applied, the number of matrix elements that should be calculated is approximately or even equal to the number $N$ of magnetic moments of the periodic cell. The usual required precision of the MDEs is high, about $10^{-6} \mathrm{eV} / \mathrm{cell}$, and the computation of the matrices to obtain MDEs with that precision is very expensive and hence, the application of these symmetries reduces drastically the computing time of the calculation of the MDEs.

This paper is organized as follows. Section 2 is devoted to the theory of the magnetostatic dipolar interaction energy of a lattice of magnetic moments or dipoles. Section 3 is devoted to explain the analysis of the symmetries to reduce the computation time of the MDE. The last section is the discussion of the computation timing results of the calculations of the MDE of $\mathrm{Ni}$ fcc nanowires up to 31500 magnetic moment in the periodic cell, using and not using the symmetries.

\section{Theory of the Magnetostatic dipolar energy of a lattice of magnetic moments}

\subsection{Magnetostatic dipolar energy of a lattice of magnetic moments}

The magnetostatic dipolar energy of a lattice of magnetic moments consists on the summation of the magnetic dipolar interaction energies between the different pairs of the magnetic moments of the lattice. This summation is given 
by:

$$
\begin{aligned}
E_{d}= & \frac{1}{2} \frac{\mu_{0}}{4 \pi} \sum_{i} \sum_{j} \sum_{n}\left(\frac{\vec{m}_{i} \cdot \vec{m}_{j}}{\left|\vec{R}_{n}+\vec{i}-\vec{j}\right|^{3}}\right. \\
& \left.-\frac{3\left(\vec{m}_{i} \cdot\left(\vec{R}_{n}+\vec{i}-\vec{j}\right)\right)\left(\vec{m}_{j} \cdot\left(\vec{R}_{n}+\vec{i}-\vec{j}\right)\right)}{\left|\vec{R}_{n}+\vec{i}-\vec{j}\right|^{5}}\right),
\end{aligned}
$$

where $i$ and $j$ denote the atoms in the cell, $\vec{i}$ is the position of atom $i$ in the cell, $\vec{m}_{i}$ is the magnetic moment of atom $i$ and the vector $\vec{R}_{n}+\vec{i}-\vec{j}$ connects the magnetic moments $\vec{m}_{i}$ and $\vec{m}_{j}$, located at $\vec{R}_{n}+\vec{i}$ and $\vec{j}$, respectively. $\vec{R}_{n}$ is a lattice site: $\vec{R}_{n}=n_{a} \vec{a}+n_{b} \vec{b}+n_{c} \vec{c}$ and $n$ stands for $n=\left(n_{a}, n_{b}, n_{c}\right)$. The sum runs over all the lattice sites $\vec{R}_{n}$ except over that for which the denominator in Eq. 1 is zero.

If all the magnetic moments $\vec{m}_{i}$ and $\vec{m}_{j}$ of the cell are parallel to the direction $\widehat{n}$, i.e., it is a ferromagnetic system, then $\vec{m}_{i}=m_{i} \widehat{n}$, with $i=1-N$, and the magnetic dipolar energy is given by:

$$
E_{d}(\widehat{n})=\frac{1}{2} \frac{\mu_{0}}{4 \pi} \sum_{i} \sum_{j} m_{i} m_{j} M_{i j},
$$

where the quantities $M_{i j}$ are called the ferromagnetic dipolar Madelung constants and are given by

$$
M_{i j}=\sum_{n}\left(\frac{1}{\left|\vec{R}_{n}+\vec{i}-\vec{j}\right|^{3}}-\frac{3\left(\widehat{n} \cdot\left(\vec{R}_{n}+\vec{i}-\vec{j}\right)\right)^{2}}{\left|\vec{R}_{n}+\vec{i}-\vec{j}\right|^{5}}\right) .
$$

These constants can be further developed, taking into account the angle $\theta_{n i j}^{\prime}$ between the magnetic moments and the vector $\vec{R}_{n}+\vec{i}-\vec{j}$ :

$$
M_{i j}=\sum_{n} \frac{1-3\left(\cos \theta_{n i j}^{\prime}\right)^{2}}{\left|\vec{R}_{n}+\vec{i}-\vec{j}\right|^{3}},
$$

where the cosine of the angle $\theta_{n i j}^{\prime}$ is given by:

$$
\cos \theta_{n i j}^{\prime}=\frac{\widehat{n} \cdot\left(\vec{R}_{n}+\vec{i}-\vec{j}\right)}{\left|\vec{R}_{n}+\vec{i}-\vec{j}\right|} .
$$

The magnetic moment, the vector $\vec{R}_{n}+\vec{i}-\vec{j}$ and the angle $\theta_{n i j}^{\prime}$ are depicted in Fig. 1).

Using the complex spherical harmonic for $l=2$ and $m=0, Y_{2,0}^{\text {complex }}=\sqrt{\frac{5}{16 \pi}}\left(3 \cos ^{2} \theta-1\right)$ [12], [13], the Madelung constants are written as:

$$
M_{i j}=-\sqrt{\frac{16 \pi}{5}} \sum_{n} \frac{Y_{2,0}^{\text {complex }}\left(\theta_{n i j}^{\prime}, \phi_{n i j}^{\prime}\right)}{\left|\vec{R}_{n}+\vec{i}-\vec{j}\right|^{3}} .
$$

The complex spherical harmonic $Y_{2,0}^{\text {complex }}\left(\theta_{n i j}^{\prime}, \phi_{n i j}^{\prime}\right)$ can be written as:

$$
\begin{aligned}
& Y_{2,0}^{\text {complex }}\left(\theta_{n i j}^{\prime}, \phi_{n i j}^{\prime}\right)= \\
& \sum_{m=-2}^{2} D_{2, m, 0}(\alpha, \beta, \gamma) Y_{2, m}^{\text {complex }}\left(\theta_{n i j}, \phi_{n i j}\right),
\end{aligned}
$$

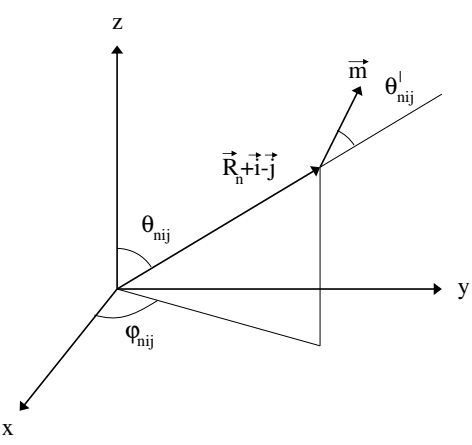

Figure 1. Vector $\vec{R}_{n}+\vec{i}-\vec{j}$, the spherical angles $\theta_{n i j}$ and $\phi_{n i j}$ of this vector with respect to the Cartesian reference system, the magnetic moment $\vec{m}$ and the spherical angle $\theta_{n i j}^{\prime}$ between the vectors $\vec{R}_{n}+\vec{i}-\vec{j}$ and $\vec{m}$. The magnetic moment $\vec{m}=m \widehat{n}$.

where $\alpha, \beta$ and $\gamma$ are the Euler angles that define the direction of the magnetic moments with respect to a Cartesian reference system, $D_{2, m, 0}$ are the Wigner rotation matrix elements [14], [15], and $\theta_{n i j}$ and $\phi_{n i j}$ are the spherical angles of the vector $\vec{R}_{n}+\vec{i}-\vec{j}$ with respect to the Cartesian reference system (See Fig. 1).

Inserting Eq. 7 into Eq. 6, the Madelung constants turn into:

$$
\begin{aligned}
& M_{i j}=-\sqrt{\frac{16 \pi}{5}} \sum_{m=-2}^{2} D_{2, m, 0}(\alpha, \beta, \gamma) \\
& \sum_{n} \frac{Y_{2, m}^{\text {complex }}\left(\theta_{n i j}, \phi_{n i j}\right)}{\left|\vec{R}_{n}+\vec{i}-\vec{j}\right|^{3}} .
\end{aligned}
$$

If the magnetic moments are in units of the Bohr magneton $\mu_{B}$, then:

$$
E_{d}(\widehat{n})=\frac{\mu_{B}^{2}}{2} \frac{\mu_{0}}{4 \pi} \sum_{i} \sum_{j} m_{i} m_{j} M_{i j} .
$$

The quantity $\mu_{B}^{2} \mu_{0} / 8 \pi$ is equal to $1 / c^{2}$ in atomic Rydberg units. Therefore, the magnetic dipolar energy in atomic Rydberg units is given by:

$$
E_{d}(\widehat{n})=\frac{1}{c^{2}} \sum_{i} \sum_{j} m_{i} m_{j} M_{i j}
$$

The Madelung constants $M_{i j}$ can be written as a combination of real spherical harmonics, using the relationship between the real and complex spherical harmonics (See Eq. 27 in the Appendix A) [12], [13]:

$$
\begin{aligned}
& M_{i j}=k\left[D_{2,0,0} \sum_{n} \frac{Y_{2,0}^{r e a l}\left(\theta_{n i j}, \phi_{n i j}\right)}{\left|\vec{R}_{n}+\vec{i}-\vec{j}\right|^{3}}+\right. \\
& \sum_{m=1}^{2} D_{2, m, 0} \sum_{n} \frac{Y_{2, m}^{r e a l}\left(\theta_{n i j}, \phi_{n i j}\right)+i Y_{2,-m}^{r e a l}\left(\theta_{n i j}, \phi_{n i j}\right)}{\sqrt{2}(-1)^{m}\left|\vec{R}_{n}+\vec{i}-\vec{j}\right|^{3}}+ \\
& \left.\sum_{m=1}^{2} D_{2,-m, 0} \sum_{n} \frac{Y_{2, m}^{r e a l}\left(\theta_{n i j}, \phi_{n i j}\right)-i Y_{2,-m}^{r e a l}\left(\theta_{n i j}, \phi_{n i j}\right)}{\sqrt{2}\left|\vec{R}_{n}+\vec{i}-\vec{j}\right|^{3}}\right],
\end{aligned}
$$


where $k=-\sqrt{\frac{16 \pi}{5}}$. Let's define the matrix elements $S_{m}(i, j)$ :

$$
S_{m}(i, j)=\sum_{n} \frac{Y_{2, m}^{r e a l}\left(\theta_{n i j}, \phi_{n i j}\right)}{\left|\vec{R}_{n}+\vec{i}-\vec{j}\right|^{3}}
$$

with $m=-2,-1,0,1,2$. Using Eq. 11 and the quantities $S_{m}(i, j)$ defined in Eq. 12 and with some algebra calculations, the Madelung constants can be written as:

$$
\begin{array}{r}
M_{i j}=k\left[S_{0}(i, j) D_{2,0,0}+\frac{S_{1}(i, j)}{\sqrt{2}}\left(-D_{2,1,0}+D_{2,-1,0}\right)+\right. \\
\frac{i S_{-1}(i, j)}{\sqrt{2}}\left(-D_{2,1,0}-D_{2,-1,0}\right)+\frac{S_{2}(i, j)}{\sqrt{2}}\left(D_{2,2,0}+D_{2,-2,0}\right)+ \\
\left.\frac{i S_{-2}(i, j)}{\sqrt{2}}\left(D_{2,2,0}-D_{2,-2,0}\right)\right]
\end{array}
$$

The Wigner rotation matrix elements have some properties [14], [15] that can be used to simplify the Madelung constants $M_{i j}$ (See Appendix B): $D_{2,-1,0}=-D_{2,1,0}^{*}$ and $D_{2,-2,0}=D_{2,2,0}^{*}$. Taking into account these properties and Eq. 13, and with some additional algebra, the Madelung constants $M_{i j}$ can be finally written as:

$$
\begin{aligned}
& M_{i j}=k\left[S_{0}(i, j) D_{2,0,0}-S_{1}(i, j) \sqrt{2} \operatorname{Real}\left(D_{2,1,0}\right)\right. \\
& +S_{-1}(i, j) \sqrt{2} \operatorname{Imag}\left(D_{2,1,0}\right)+S_{2}(i, j) \sqrt{2} \operatorname{Real}\left(D_{2,2,0}\right) \\
& \left.-S_{-2}(i, j) \sqrt{2} \operatorname{Imag}\left(D_{2,2,0}\right)\right] \cdot \quad(14)
\end{aligned}
$$

The MDE is calculated using Eqs. 10, 12 and 14. The matrix elements $S_{m}(i, j)$ in Eq. 12 are computed by means of the Ewald summation method [8], [9].

\subsection{Magnetostatic dipolar anisotropy energy}

The magnetostatic dipolar anisotropy energy, MDAE, is the difference between the magnetostatic dipolar energies for two different magnetization directions. For instance, in the case of magnetizations $\vec{M}$ parallel and perpendicular to the c-axis $\widehat{c}$ of a layered system (this axis is perpendicular to the plane of the layers), the magnetostatic dipolar anisotropy energy is given by:

$$
M D A E(\|, \perp)=E_{d}(\widehat{n} \| \widehat{c})-E_{d}(\widehat{n} \perp \widehat{c}),
$$

where $\widehat{n}=\vec{M} / M$ is a unitary vector along the magnetization, $\widehat{c}$ is a unitary vector along the c-axis and the magnetostatic dipolar energies $E_{d}$ 's are given by Eq. 10, with the corresponding orientations of the magnetizations.

In the study of the MDAE of magnetic layered systems, the directions of interest are the axis perpendicular and parallel to the plane of the layers. The parallel axis is not well defined, because there are many axes lying in the plane of the layers. Usually the perpendicular axis is denoted as the $z$ axis and the parallel axis could be any axis lying in the $x y$ plane. This is the convention that we have followed in this paper, unless otherwise noted.

\section{Analysis of the Symmetries of the $S$ matrices}

The magnetostatic dipolar energy, $\mathrm{MDE}$, is a long-range interaction and hence, in a periodic system of $N$ magnetic moments, the interaction of each magnetic moment $i$ with every other magnetic moment $j$ must be calculated. The MDE of periodic magnetic systems is calculated by means of the Ewald's lattice summation method [8], [9], [10], [11]. This method is used to calculate the five matrix elements $S_{m}(i, j) \quad(\mathrm{m}=-2,-1,0,1,2)$, Eq. 12 , related to the magnetostatic dipolar interaction between the magnetic moments $i$ and $j$ in all the cells (the real cell and the replicated cells). These five matrix elements are then, used to calculate the matrix element $M_{i j}$ through Eq. 14. The calculation of the MDE, Eq. 2, of periodic magnetic systems using the traditional Ewald method is an $O\left(N^{2}\right)$ task, because there are $N(N-1) / 2+1$ different matrix elements $M_{i j}$ in the summation of that equation, or six times $N(N-1) / 2+1$ different matrix elements $S_{m}(i, j)$, as can be noticed in Eq. 14. A detailed analysis of the time complexity of the traditional Ewald method was published by Petersen [10] and Wang and Holm [11].

Each Madelung constant $M_{i j}$ (or equivalently each of the five $S_{m}(i, j)$ matrix elements) is a summation over the infinite number of lattice sites $\vec{R}_{n}$ of the magnetic periodic system (See Eq. 3). The summation to calculate $M_{i j}$ in Eq. 3 is obtained by applying cutoff distances in real and reciprocal spaces and it converges rapidly. The MDEs and MDAEs are very small energies. The Madelung constants $M_{i j}$ must be calculated with enough precision to ensure MDEs, and especially MDAEs, with a precision of at least $10^{-6} \mathrm{eV} / \mathrm{cell}$. The MDAE is the difference between two MDEs and both must be enough accurate, to obtain the MDAE as an accurate difference, without effects due to the compensation of errors.

A strategy to reduce the computation time of the calculation of the MDE with high precision, without changing the cutoff distances, consists on using the symmetries of the periodic magnetic system. To use those symmetries, one should consider and analyze the $\mathrm{S}$ matrices in more detail. The S matrices of the Ewald method applied to the calculation of the magnetostatic dipolar energy are given by Eq. 12, where $Y_{2, m}^{r e a l}$ is a real spherical harmonic of $l=2$ and $m=-2,-1,0,1,2, \vec{i}$ and $\vec{j}$ are the positions of the $i$ and $j$ atoms in the cell, respectively, and $\vec{R}_{n}$ is a Bravais lattice vector or lattice site, i.e., $\vec{R}_{n}=n_{a} \vec{a}+n_{b} \vec{b}+n_{c} \vec{c}$, with $\vec{a}, \vec{b}$ and $\vec{c}$ equal to the lattice vectors of the cell, and $n_{a}, n_{b}$ and $n_{c}$ are integer numbers. The position vector of atom $i$ is given by $\vec{i}=\left(x_{i}, y_{i}, z_{i}\right)$.

The real spherical harmonics in the definition of $S_{m}(i, j)$ depend on $\theta_{n i j}$ and $\phi_{n i j}$ and are obtained from Eq. 29 by making the following replacements in that equation: $x$ replaced by $X_{n}+x_{i}-x_{j}$,y replaced by $Y_{n}+y_{i}-z_{j}$ and $z$ replaced by $Z_{n}+z_{i}-z_{j}$ and $r=\left|\vec{R}_{n}+\vec{i}-\vec{j}\right|$.

The $\mathrm{S}$ matrices are symmetric, i.e., $S_{m}(i, j)=S_{m}(j, i)$. This is taken into account in all the calculations and this does not depend on the type of Bravais lattice cell, nor in 
the values of the vectors $\vec{i}-\vec{j}$ of the basis atoms of the cell.

If the vectors $\vec{i}-\vec{j}$ and $\vec{k}-\vec{l}$ of the basis atoms of the cell and the Bravais lattice cell satisfy certain conditions, then the matrix elements $S_{m}(i, j)$ are equal to $\pm S_{m}(k, l)$, with $m=-2,-1,0,1,2$. These symmetries or conditions allow us to reduce the number of matrix elements that should be calculated.

The general symmetry or condition that must be satisfied is as follows: If any vector $\vec{R}_{n}+\vec{i}-\vec{j}$ is equal to the vector $\vec{T}+\vec{k}-\vec{l}$, such as

$$
\frac{Y_{2, m}^{\text {real }}\left(\theta_{n i j}, \phi_{n i j}\right)}{\left|\vec{R}_{n}+\vec{i}-\vec{j}\right|^{3}}= \pm \frac{Y_{2, m}^{\text {real }}\left(\theta_{t k l}, \phi_{t k l}\right)}{|\vec{T}+\vec{k}-\vec{l}|^{3}}
$$

where the vector $\vec{T}$ should be a Bravais lattice vector, i.e., $\vec{T}=\vec{R}_{p}=p_{a} \vec{a}+p_{b} \vec{b}+p_{c} \vec{c}$, with $p_{a}, p_{b}$ and $p_{c}$ being integer numbers, then $S_{m}(i, j) \pm S_{m}(k, l)$.

If $\vec{T}=\vec{R}_{p}$, then Eq. 16 implies a reordering of the sums in the summation that defines $S_{m}(i, j)$, Eq. 12, but the value of the summation does not change, except for a sign in some cases, depending on the value of $m$. On the contrary, if $\vec{T}$ is not a Bravais lattice vector, then Eq. 16 is not satisfied and the absolute value of the summation in Eq. 12 changes.

The Eq. 16 will be satisfied depending on the values of $\vec{i}-\vec{j}$ and $\vec{k}-\vec{l}$, and on the type of Bravais lattice cell. There are at least eight symmetries or conditions of $\vec{i}-\vec{j}$ and $\vec{k}-\vec{l}$ that could lead to the fulfillment of Eq. 16 . The first and second conditions satisfy Eq. 16 for any type of Bravais lattice cell:

1) If $\vec{i}-\vec{j}$ is equal to $\vec{k}-\vec{l}$ then $S_{m}(k, l)=S_{m}(i, j)$ for any value of $m$ :

If $\vec{i}-\vec{j}=\vec{k}-\vec{l}$, then $\vec{T}+\vec{k}-\vec{l}=\vec{R}_{n}+\vec{k}-\vec{l}=\vec{R}_{n}+\vec{i}-\vec{j}$, $\theta_{t k l}=\theta_{n k l}=\theta_{n i j}$ and $\phi_{t k l}=\phi_{n k l}=\phi_{n i j}$, which implies that $S_{m}(i, j)=S_{m}(k, l)$.

An implication of this symmetry is that the interaction of atom $i$ of the cell with all the atoms $i$ of the replicated cells, is the same that the interaction of atom $j$ with all the atoms $j$ of the replicated cells. This, in turn, means that all the elements of the diagonal of the corresponding $S_{m}$ matrices are identical: $S_{m}(i, i)=S_{m}(1,1)$, with $m=-2,-1,0,1,2$. Hence, to calculate the elements of the diagonal of $S_{m}$ we need to calculate only one element, $S_{m}(1,1)$. Notice that $S_{0}(1,1)$ is different from $S_{2}(1,1)$ and so on for $m=-2,-1,0,1,2$. Only five matrix elements are necessary to calculate the corresponding diagonals of the five $S_{m}$ matrices. The fact that $S_{m}(i, i)=S_{m}(1,1)$ for any value of $i$ is applied in all the calculations, not only on the calculations that use the symmetries of the periodic magnetic system.

2) If $\vec{i}-\vec{j}$ is equal to $-(\vec{k}-\vec{l})$ then $S_{m}(k, l)=S_{m}(i, j)$ for any value of $m$ :

This symmetry comes from the fact that the $\mathrm{S}$ matrices are symmetric: If $\vec{i}-\vec{j}=-\vec{k}-\vec{l} \rightarrow \vec{R}_{n}+\vec{i}-\vec{j}=\vec{R}_{n}+\vec{l}-\vec{k}$, which means that $S_{m}(i, j)=S_{m}(l, k)$. The matrices $S_{m}$ are symmetric matrices, therefore $S_{m}(l, k)=S_{m}(k, l)$, and $S_{m}(i, j)=S_{m}(k, l)$.

The following six symmetries or conditions do not fulfill Eq. 16 for all the Bravais lattice cells. If the cell belongs to the following group of Bravais lattice cells: simple cubic, fcc, bcc, simple tetragonal and simple orthorhombic, then $\vec{T}$ will be a Bravais lattice vector $\vec{R}_{p}$ of the cell and the conditions 3-8 will satisfy Eq. 16.

3) If $x_{i}-x_{j}=-\left(x_{k}-x_{l}\right), y_{i}-y_{j}=y_{k}-y_{l}$ and $z_{i}-z_{j}=z_{k}-z_{l}$, then, taking into account the dependence on $x_{i}-x_{j}$ and $x_{k}-x_{l}$ of $Y_{2, m}$, we find:

$$
\begin{aligned}
& S_{-2}(k, l)=-S_{-2}(i, j) \\
& S_{-1}(k, l)=S_{-1}(i, j) \\
& S_{0}(k, l)=S_{0}(i, j) \\
& S_{1}(k, l)=-S_{1}(i, j) \\
& S_{2}(k, l)=S_{2}(i, j) .
\end{aligned}
$$

The Eqs. 17 can be proved as follows. If $x_{i}-x_{j}=-$ $\left(x_{k}-x_{l}\right), y_{i}-y_{j}=y_{k}-y_{l}$ and $z_{i}-z_{j}=z_{k}-z_{l}$, then:

$$
X_{n i j}=X_{n}+x_{i}-x_{j}=X_{n}-\left(x_{k}-x_{l}\right)=X_{n}+x_{l}-x_{k}
$$

$Y_{n i j}=Y_{n}+y_{i}-z_{j}=Y_{n}+\left(y_{k}-y_{l}\right)=-\left(-Y_{n}+x_{l}-x_{k}\right)$

$Z_{n i j}=Z_{n}+z_{i}-z_{j}=Z_{n}+\left(x_{k}-z_{l}\right)=-\left(-Z_{n}+z_{l}-z_{k}\right)$.

These three equations mean that $\left|\vec{R}_{n}+\vec{i}-\vec{j}\right|=$ $|\vec{T}+\vec{l}-\vec{k}|, Y_{2 m}\left(\theta_{n i j}, \phi_{n i j}\right)= \pm Y_{2 m}\left(\theta_{t l k}, \phi_{t l k}\right)$. If $\vec{T}=$ $\left(X_{n},-Y_{n},-Z_{n}\right)$ is a Bravais lattice vector, let's say, $\vec{T}=$ $\vec{R}_{p}$, then $S_{m}(i, j)= \pm S_{m}(l, k)=S_{m}(k, l)$ and $\mid \vec{R}_{n}+\vec{i}-$ $\vec{j}|=| \vec{T}+\vec{l}-\vec{k}|=| \vec{R}_{p}+\vec{l}-\vec{k} \mid$. The sign in front of $Y_{2 m}$ is also the sign in front of $S_{m}(k, l)$. The sign depends on the value of $m$.

The vector $\vec{T}$ is the Bravais lattice vector $\vec{R}_{p}$. Hence, the coordinates above are equal to:

$$
\begin{gathered}
X_{n i j}=X_{n}+x_{i}-x_{j}=X_{n}-\left(x_{k}-x_{l}\right)=X_{n}+x_{l}-x_{k}= \\
X_{p}+x_{l}-x_{k}=X_{p l k} \\
Y_{n i j}=Y_{n}+y_{i}-z_{j}=Y_{n}+\left(y_{k}-y_{l}\right)=-\left(-Y_{n}+x_{l}-x_{k}\right)= \\
-\left(Y_{p}+y_{l}-y_{k}\right)=-Y_{p l k} \\
Z_{n i j}=Z_{n}+z_{i}-z_{j}=Z_{n}+\left(x_{k}-z_{l}\right)=-\left(-Z_{n}+z_{l}-z_{k}\right)= \\
-\left(Z_{p}+z_{l}-z_{k}\right)=-Z_{p l k} .
\end{gathered}
$$

With these equations we can calculate the real spherical harmonics:

$$
\begin{gathered}
Y_{2,-2}^{r e a l}\left(\theta_{n i j}, \phi_{n i j}\right)=C_{2} X_{n i j} Y_{n i j} / R_{n i j}^{2}= \\
-C_{2} X_{p l k} Y_{p l k} / R_{p l k}^{2}=-Y_{2,-2}^{r e a l}\left(\theta_{p l k}, \phi_{p l k}\right) \\
Y_{2,-1}^{r e a l}\left(\theta_{n i j}, \phi_{n i j}\right)=C_{1} Y_{n i j} Z_{n i j} / R_{n i j}^{2}= \\
C_{1} Y_{p l k} Z_{p l k} / R_{p l k}^{2}=Y_{2,-1}^{r e a l}\left(\theta_{p l k}, \phi_{p l k}\right) \\
Y_{2,0}^{r e a l}\left(\theta_{n i j}, \phi_{n i j}\right)=C_{0}\left(3 Z_{n i j}^{2}-R_{n i j}^{2}\right) / R_{n i j}^{2}= \\
C_{0}\left(3 Z_{p l k}^{2}-R_{p l k}^{2}\right) / R_{p l k}^{2}=Y_{2,0}^{r e a l}\left(\theta_{p l k}, \phi_{p l k}\right) \\
Y_{2,1}^{r e a l}\left(\theta_{n i j}, \phi_{n i j}\right)=C_{1} X_{n i j} Z_{n i j} / R_{n i j}^{2}= \\
-C_{1} X_{p l k} Z_{p l k} / R_{p l k}^{2}=-Y_{2,1}^{r e a l}\left(\theta_{p l k}, \phi_{p l k}\right) \\
Y_{2,2}^{r e a l}\left(\theta_{n i j}, \phi_{n i j}\right)=C_{2}\left(X_{n i j}^{2}-Y_{n i j}^{2}\right) / R_{n i j}^{2}= \\
C_{2}\left(X_{p l k}^{2}-Y_{p l k}^{2}\right) / R_{p l k}^{2}=Y_{2,2}^{r e a l}\left(\theta_{p l k}, \phi_{p l k}\right)
\end{gathered}
$$


where $R_{n i j}=\left|\vec{R}_{n}+\vec{i}-\vec{j}\right|, R_{p l k}=\left|\vec{R}_{p}+\vec{l}-\vec{k}\right|$, and the constants are given by $C_{0}=\sqrt{\frac{5}{16 \pi}}, C_{1}=\sqrt{\frac{15}{4 \pi}}$ and $C_{2}=\sqrt{\frac{15}{16 \pi}}$.

Considering Eq. 12 and 20, $\left|\vec{R}_{n}+\vec{i}-\vec{j}\right|=\left|\vec{R}_{p}+\vec{l}-\vec{k}\right|$ and $S_{m}(l, k)=S_{m}(k, l)$ for any value of $m$, we obtain the matrix elements in Eqs. 17.

4) If $x_{i}-x_{j}=x_{k}-x_{l}, y_{i}-y_{j}=-\left(y_{k}-y_{l}\right)$ and $z_{i}-z_{j}=z_{k}-z_{l}$, then, taking into account the dependence on $y_{i}-y_{j}$ and $y_{k}-y_{l}$ of $Y_{2, m}$ :

$$
\begin{aligned}
& S_{-2}(k, l)=-S_{-2}(i, j) \\
& S_{-1}(k, l)=-S_{-1}(i, j) \\
& S_{0}(k, l)=S_{0}(i, j) \\
& S_{1}(k, l)=S_{1}(i, j) \\
& S_{2}(k, l)=S_{2}(i, j) .
\end{aligned}
$$

5) If $x_{i}-x_{j}=x_{k}-x_{l}, y_{i}-y_{j}=y_{k}-y_{l}$ and $z_{i}-z_{j}=-$ $\left(z_{k}-z_{l}\right)$, then, taking into account the dependence on $z_{i}-z_{j}$ and $z_{k}-z_{l}$ of $Y_{2, m}$ :

$$
\begin{aligned}
& S_{-2}(k, l)=S_{-2}(i, j) \\
& S_{-1}(k, l)=-S_{-1}(i, j) \\
& S_{0}(k, l)=S_{0}(i, j) \\
& S_{1}(k, l)=-S_{1}(i, j) \\
& S_{2}(k, l)=S_{2}(i, j) .
\end{aligned}
$$

6) If $x_{i}-x_{j}=-\left(x_{k}-x_{l}\right), y_{i}-y_{j}=-\left(y_{k}-y_{l}\right)$ and $z_{i}-$ $z_{j}=z_{k}-z_{l}$, then, taking into account the dependence on $x_{i}-x_{j}, y_{i}-y_{j}, x_{k}-x_{l}$ and $y_{k}-y_{l}$ of $Y_{2, m}$ :

$$
\begin{aligned}
& S_{-2}(k, l)=S_{-2}(i, j) \\
& S_{-1}(k, l)=-S_{-1}(i, j) \\
& S_{0}(k, l)=S_{0}(i, j) \\
& S_{1}(k, l)=-S_{1}(i, j) \\
& S_{2}(k, l)=S_{2}(i, j) .
\end{aligned}
$$

7) If $x_{i}-x_{j}=-\left(x_{k}-x_{l}\right), y_{i}-y_{j}=y_{k}-y_{l}$ and $z_{i}-z_{j}=-$ $\left(z_{k}-z_{l}\right)$, then, taking into account the dependence on $x_{i}-x_{j}$, $z_{i}-z_{j}, x_{k}-x_{l}$ and $z_{k}-z_{l}$ of $Y_{2, m}$ :

$$
\begin{aligned}
& S_{-2}(k, l)=-S_{-2}(i, j) \\
& S_{-1}(k, l)=-S_{-1}(i, j) \\
& S_{0}(k, l)=S_{0}(i, j) \\
& S_{1}(k, l)=S_{1}(i, j) \\
& S_{2}(k, l)=S_{2}(i, j) .
\end{aligned}
$$

8) If $x_{i}-x_{j}=x_{k}-x_{l}, y_{i}-y_{j}=-\left(y_{k}-y_{l}\right)$ and $z_{i}-z_{j}=-$ $\left(z_{k}-z_{l}\right)$, then, taking into account the dependence on $y_{i}-y_{j}$, $z_{i}-z_{j}, y_{k}-y_{l}$ and $z_{k}-z_{l}$ of $Y_{2, m}$ :

$$
\begin{aligned}
& S_{-2}(k, l)=-S_{-2}(i, j) \\
& S_{-1}(k, l)=S_{-1}(i, j) \\
& S_{0}(k, l)=S_{0}(i, j) \\
& S_{1}(k, l)=-S_{1}(i, j) \\
& S_{2}(k, l)=S_{2}(i, j) .
\end{aligned}
$$

The quantities $X_{n}, Y_{n}$ and $Z_{n}$ in the equations are inside a summation over an infinite number of lattice vectors $\vec{R}_{n}$. If the vector $\vec{T}$ is also a Bravais lattice vector, i.e., $\vec{T}=$ $\left(X_{n},-Y_{n},-Z_{n}\right)=\vec{R}_{p}=\left(X_{p}, Y_{p}, Z_{p}\right)=p_{a} \vec{a}+p_{b} \vec{b}+p_{c} \vec{c}$, then the order of the sums in the summation is changed. The result of the sums, however, does not change by changing the order of the sums. If $\vec{T}$ is not a Bravais lattice vector or lattice site, i.e., $\vec{T} \neq \vec{R}_{p}$, for the studied cell, then the summation changes and $S_{m}(i, j) \neq S_{m}(k, l)$.

These symmetries have been tested in the 14 Bravais lattices. The first two symmetries, 1) and 2), are satisfied by the 14 Bravais lattices. As regards to the other six symmetries, $3)-8$ ), the result depends on the type of Bravais lattice. Some lattices satisfy the six symmetries, some lattices satisfy a few symmetries and the triclinic lattice does not satisfy any of the six symmetries. The analysis of the symmetries has been tested in systems like nanowires, disordered nanowires, crystals, slabs, ribbons and multisegmented nanowires.

If the basis atoms of the cell do not satisfy the conditions 3)-8), then the number of S matrix elements that should be calculated will not be reduced, even if the cell is one of the lattice that satisfy the symmetries 3)-8). If some atoms satisfy the conditions 3)-8), then the number of $\mathrm{S}$ matrix elements will be reduced according to Eqs. 17,21-25. The more basis atoms that satisfy conditions $3-8$, the lesser the number of S matrix elements that should be calculated.

If the periodic system satisfy the conditions 3)-8), then many matrix elements are identical to other elements due to symmetry reasons, or differ only in the sign of the matrix elements. It is not necessary to calculate all of them. Only one of the identical elements should be calculated. Hence, using the symmetries of the periodic magnetic system, the number of $\mathrm{S}$ matrix elements that should be calculated is reduced drastically, and hence also the computation time is reduced.

The algorithm to analyze the above symmetries and to determine which elements of the $\mathrm{S}$ matrix should be calculated and which should not be calculated, consists on a conditioned comparison of the pairs of vectors $\vec{i}-\vec{j}$ and $\vec{k}-\vec{l}$ of the basis atoms of the cell. The pairs that satisfy some of the symmetries or conditions are not compared anymore. This type of conditioned comparison is an $O\left(N^{3}\right)$ task. This comparison is valid for any type of lattice. The application of the symmetries has been tested in the 14 Bravais lattices and in the following periodic magnetic systems: crystals, nanowires, multisegmented nanowires, ribbons, slabs, nanotubes and spheres of magnetic moments, obtaining a significant decrease of the computation time. In the next section, the computation timing results obtained for a particular case of large periodic magnetic systems, with fundamental and 
technological interest, $\mathrm{Ni}$ fcc nanowires, are analyzed and explained.

\section{Reduction of the Computation Time of the Calculation of the MDE of Ni fcc Nanowires}

A Ni fcc nanowire is a nanowire of finite radius, composed by $\mathrm{Ni}$ atoms with the structure of bulk $\mathrm{Ni}$ fcc (See Fig. 2). The periodic cell that contains a $\mathrm{Ni}$ fcc nanowire of radius $r$ consists on a tetragonal cell with lattice parameters $s, s$ and $h$, where $s=2 r+i, h$ is the height of the nanowire in the cell and $i$ is the distance between the external walls of the nanowires of adjacent cells. The height $h$ and the distance $i$ are kept fixed in all the calculations to $h=a$ and $i=10 a$, where the quantity $a$ is the experimental value of the lattice parameter of bulk $\mathrm{Ni}$ fcc, $3.52 \AA$. The tetragonal cell and the basis atoms are such that the nanowires are infinite along the main axis. Nanowires with a radius between $r=a$ a $r=50 a$ were studied. Each $\mathrm{Ni}$ atom has a magnetic moment. The computation timing experiments of the MDE of $\mathrm{Ni}$ fcc nanowires have been carried out in a computer with a $2.50 \mathrm{GHz} \operatorname{Intel}(\mathrm{R}) \mathrm{Xeon}(\mathrm{R})$ E5-2640 processor.

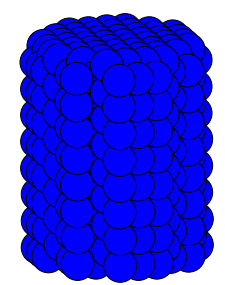

Figure 2. Depiction of a Ni fcc nanowire with radius of $3 a, a=3.52 \AA$.

Fig. 3 shows the MDE along $\mathrm{z}$ axis, $\operatorname{MDE}(\mathrm{z})$ and the magnetostatic dipolar anisotropy energy between $\mathrm{z}$ and $\mathrm{x}$ axis, $\operatorname{MDAE}(\mathrm{z}, \mathrm{x})=\operatorname{MDE}(\mathrm{z})-\operatorname{MDE}(\mathrm{x})$, of a $\mathrm{Ni}$ fcc nanowire of radius $30 a$ as a function of the reciprocal space cutoff distance, $g_{c}$. The periodic cell of this nanowire has 5025 atoms. The real space cutoff distance, $r_{c}$, was kept fixed to 20a. The MDE and MDAE decrease as $g_{c}$ increases and converge towards some values. To obtain MDE and MDAE with the desired precision of $10^{-6} \mathrm{eV} /$ cell, the reciprocal space cutoff distance should be at least 9/a radians/ $\AA$ for this nanowire. We have studied Ni fcc nanowires of different sizes and we have found the same behaviour of MDE and MDAE vs $g_{c}$ and also similar minimum values of $g_{c}$ distances, 8/a-9/a radians/ $\AA$, to obtain MDEs and MDAEs with $10^{-6} \mathrm{eV} /$ cell of precision. After running several tests, values of $r_{c}=38 a$ and $g_{c}=9 / a$ were used in all the calculations of $\mathrm{Ni}$ fcc nanowires, to obtain MDEs and MDAEs with a precision of $10^{-6} \mathrm{eV} / \mathrm{cell}$, the usual required precision for MDEs and MDAEs.

The computing times of the calculation of the MDE of a Ni fcc nanowire with a radius of $30 a$, as a function of $g_{c}$ with and without applying the symmetries, are plotted in Fig. 4. The computing time of the calculations done without

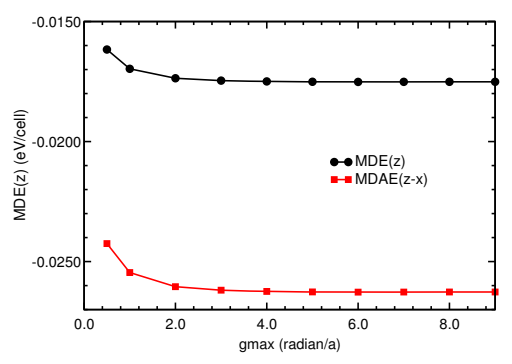

Figure 3. $\operatorname{MDE}(\mathrm{z})$ and $\operatorname{MDAE}(\mathrm{z}, \mathrm{x})=\operatorname{MDE}(\mathrm{z})-\operatorname{MDE}(\mathrm{x})$ vs reciprocal space cutoff distance of a Ni fcc nanowire with radius of $30 a$. Real space cutoff distance $=20 a$.

using the symmetries grows quadratically with $g_{c}$, while the computing time of those calculations done using the symmetries grows linearly and very slowly with $g_{c}$, being almost constant with respect to $g_{c}$. The two curves cross at $g_{c}=0.8 / a$ and below the crossing point, the computation time of the calculations without using the symmetries is lower than the computation time using the symmetries. However, below the crossing point, the MDEs and MDAE have a low precision, of about $10^{-3}-10^{-4} \mathrm{eV} / \mathrm{cell}$, in the studied nanowires. It is necessary to use higher values, $g_{c}=9 / \mathrm{a}$ radians/ $\AA$, to obtain the MDEs and MDAE with the required high precision of $10^{-6} \mathrm{eV} /$ cell.

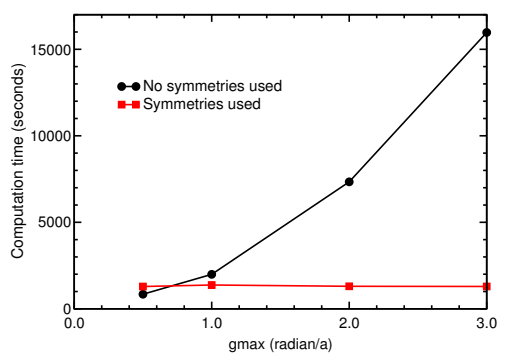

Figure 4. Computation time of the MDE of a Ni fcc nanowires with radius of $30 a$ vs reciprocal space cutoff distance. Real space cutoff distance $=20 a$.

The computation timing results using and not using the symmetries have been plotted in Fig. 5 versus the number of atoms of the Ni fcc nanowires. These are calculations of the MDE of periodic Ni fcc nanowires of increasing radius, and hence, of increasing number of atoms. It can be noticed in Fig. 5 that the reduction of the computation time is very important. For instance, the calculation of a Ni fcc nanowire of 5025 atoms takes about 27000 seconds not using the symmetries, and about 130 seconds using the symmetries, in the mentioned computer and with the same cutoff distances.

Another way to realize the reduction of the computation time is to fix the amount of the computation time of the calculations and to find out the number of atoms of the nanowires calculated in that same fixed amount of time. For instance, a calculation of a nanowire of 19000 atoms using the symmetries and another calculation of a nanowire of 2900 atoms not using the symmetry, will take approximately the same amount of time, about 6000 seconds. 


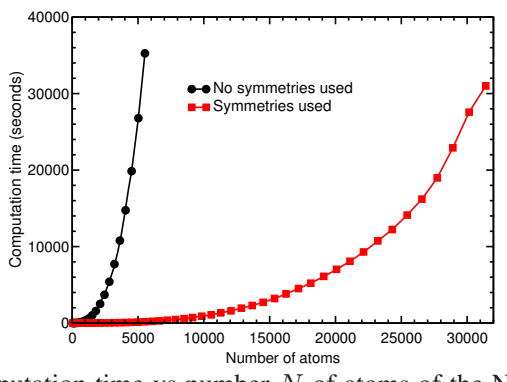

Figure 5. Computation time vs number $N$ of atoms of the $\mathrm{Ni}$ fcc wire, not using and using the symmetries of the $\mathrm{S}$ matrix.

The computation time of the calculations not using the symmetries grows faster than the computation time of the calculations using the symmetries. This can be noticed in Fig. 5. Another interesting fact is that the use of the symmetries has a much larger impact on the calculations of large systems than on the calculations of small systems: For the smallest nanowire studied without using the symmetries, the reduction factor of the computation time is about six and for the largest nanowire studied without using the symmetries, which has 5025 atoms, the reduction factor is about 200.

To analyze the dependence on $N$ of the computation time of the calculations done using the symmetries, the two main contributions to the computation time have been considered: The time to find and analyze the symmetries of the periodic magnetic system and to determine which matrix elements should be calculated, $t_{a}$, and the time to calculate the matrix elements that should be calculated, $t_{m}$. These two times are plotted in Fig. 6. The computation time $t_{a}$, is larger than $t_{m}$ and $t_{a}$ increases faster than $t_{m}$ as the number $N$ of atoms increases.

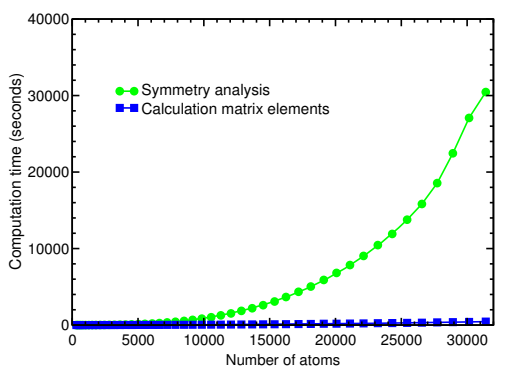

Figure 6. Computation time to analyze the symmetries, $t_{a}$, and to calculate the matrix elements, $t_{m}$, vs the number of atoms of Ni fcc nanowires, when the symmetries of the periodic magnetic system elements are used.

If the symmetries of the periodic magnetic system are not used, then $t_{m}$ is proportional to $N^{2}$ for large values of $N$. If the symmetries are used, then $t_{m}$ is proportional to $N$. To understand why $t_{m}$ is proportional to $N$ if the symmetry is used and to $N^{2}$ if the symmetries are not used, we have to further analyze $t_{m}$. The time to calculate the $\mathrm{S}$ matrix elements is proportional to the number $M$ of matrix elements: $t_{m}=a M$. If the symmetries are not used, then the number $M$ of matrix elements of $\mathrm{S}$ is not reduced and $M$ is equal to $N(N-1) / 2+1$, which means that $M$ is proportional to $N^{2}$ for large values of the number $N$ of atoms of the cell. If the symmetries are used, then $M$ is approximately equal to $N$. This fact can be noticed in Fig. 7 . The number of matrix elements that should be calculated, versus the number of atoms, when the symmetries are used, has been plotted in Fig. 7. It can be noticed in that Figure that the number of matrix elements that should be calculated is practically equal to the number of atoms $N$. For instance, the rightmost point in Fig. 7 corresponds to a Ni fcc nanowire with $N=31417$ atoms and $M=31574$ matrix elements.

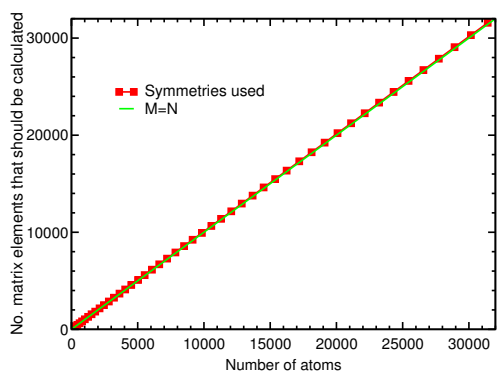

Figure 7. Number $M$ of matrix elements that should be calculated vs number $N$ of atoms of the $\mathrm{Ni}$ fcc wire, when the symmetries of the periodic magnetic system elements are used.

This dependence of the number $M$ of matrix elements on the number $N$ of magnetic moments explains the dependence on $N$ of the computation time to calculate the matrix elements: $t_{m}=b N$, when the symmetries are used, and $t_{m}=b[N(N-1) / 2+1] \approx c N^{2}$, when the symmetries are not used.

\section{Conclusions}

The symmetry properties of periodic magnetic systems of $N$ magnetic moments have been analyzed, in order to reduce the number of matrix elements that should be calculated in the traditional Ewald method utilized to calculate the MDE. The number of matrix elements of this method is $N^{2} / 2$ and hence, its time complexity is $O\left(N^{2}\right)$. It has been shown that if the periodic magnetic system has certain symmetries, then there are many matrix elements are identical to other elements, except the sign of some matrix elements. This reduces the number of matrix elements to approximately $N$, according to computation timing experiments carried out in large periodic magnetic systems, such as large Ni fcc nanowires up to 31500 magnetic moments, instead of $N^{2} / 2$, decreasing considerably the computation time of the MDE. This reduction is in contrast with the fact that the analysis of the symmetries is an $O\left(N^{3}\right)$ task, which increases the time complexity of the traditional Ewald method. The origin of this contrast is that the MDE and MDAE are very small energies and therefore, the usual required precision to calculate these energies is so high, $10^{-6} \mathrm{eV} / \mathrm{cell}$, that the calculation of the matrix elements is very expensive and, in practice, the computations carried out using the analysis of the symmetries are much faster, in spite of the larger time complexity of the analysis of the symmetries. 


\section{Acknowledgments}

This work was supported by MINECO of Spain (Grant MAT2014-54378-R), Junta de Castilla y León (Grant VA050U14) and the University of Valladolid. We acknowledge the facilities provided by Centro de Proceso de Datos - Parque Científico of the University of Valladolid.

\section{Appendix A. Complex and Real Spherical Har- monics} [13]:

The complex spherical harmonics are defined by [12],

$$
\begin{array}{r}
Y_{l,|m|}^{\text {complex }}=\Theta_{l,|m|}(\theta) e^{i|m| \phi} \\
Y_{l,-|m|}^{\text {complex }}=(-1)^{|m|} \Theta_{l,|m|}(\theta) e^{-i|m| \phi}=(-1)^{|m|} Y_{l,|m|}^{\text {complex* }}
\end{array}
$$

The complex spherical harmonics can be also written as a combination of real spherical harmonics [12], [13]:

$$
\begin{array}{r}
Y_{l, 0}^{\text {complex }}=Y_{l, 0}^{\text {real }} \\
Y_{l,|m|}^{\text {complex }}=\frac{(-1)^{|m|}}{\sqrt{2}}\left(Y_{l,|m|}^{\text {real }}+i Y_{l,-|m|}^{\text {real }}\right) \\
Y_{l,-|m|}^{\text {complex }}=\frac{1}{\sqrt{2}}\left(Y_{l,|m|}^{\text {real }}-i Y_{l,-|m|}^{\text {real }}\right)
\end{array}
$$

From Eqs. 27 we obtain the real spherical harmonics as a combination of complex spherical harmonics:

$$
\begin{array}{r}
Y_{l, 0}^{\text {real }}=Y_{l, 0}^{\text {complex }} \\
Y_{l,|m|}^{\text {real }}=\frac{1}{\sqrt{2}}\left(Y_{l,-|m|}^{\text {complex }}+(-1)^{|m|} Y_{l,|m|}^{\text {complex }}\right) \\
Y_{l,-|m|}^{\text {real }}=\frac{i}{\sqrt{2}}\left(Y_{l,-|m|}^{\text {complex }}-(-1)^{|m|} Y_{l,|m|}^{\text {complex }}\right)
\end{array}
$$

The real spherical harmonics of $l=2$ are given by [12], [13]:

$$
\begin{aligned}
Y_{2,0}^{\text {real }} & =\sqrt{\frac{5}{16 \pi}}\left(3 \cos ^{2} \theta-1\right)=\sqrt{\frac{5}{16 \pi}} \frac{3 z^{2}-x^{2}-y^{2}-z^{2}}{r^{2}} \\
Y_{2,1}^{\text {real }} & =\sqrt{\frac{15}{4 \pi}} \sin \theta \cos \theta \cos \varphi=\sqrt{\frac{15}{4 \pi}} \frac{x z}{r^{2}} \\
Y_{2,-1}^{\text {real }} & =\sqrt{\frac{15}{4 \pi}} \sin \theta \cos \theta \sin \varphi=\sqrt{\frac{15}{4 \pi}} \frac{y z}{r^{2}} \\
Y_{2,2}^{\text {real }} & =\sqrt{\frac{15}{16 \pi}} \sin ^{2} \theta \cos 2 \varphi=\sqrt{\frac{15}{16 \pi}} \frac{x^{2}-y^{2}}{r^{2}} \\
Y_{2,-2}^{\text {real }} & =\sqrt{\frac{15}{16 \pi}} \sin ^{2} \theta \sin 2 \varphi=\sqrt{\frac{15}{4 \pi}} \frac{x y}{r^{2}} .
\end{aligned}
$$

[15]:

\section{Appendix B. Some properties of the rotation matrix elements}

The Wigner rotation matrix elements are given by [14],

$$
\begin{array}{r}
D_{l, m^{\prime}, m}(\alpha, \beta, \gamma)^{*}=(-1)^{m^{\prime}-m} D_{l,-m^{\prime},-m}(\alpha, \beta, \gamma) \\
D_{l, m^{\prime}, m}(\alpha, \beta, \gamma)=e^{-i m^{\prime} \alpha} d_{l, m^{\prime}, m}(\beta) e^{-i m \gamma} \\
d_{l, m^{\prime}, m}(\beta)=(-1)^{m^{\prime}-m} d_{l,-m,-m^{\prime}}(\beta)
\end{array}
$$

If the above definition is applied to the specific cases $l=2, m^{\prime}= \pm 1, \pm 2$ and $m=0$, we obtain:

$$
\begin{array}{r}
D_{2,1,0}=e^{-i \alpha} d_{2,1,0}(\beta) \\
D_{2,-1,0}=-e^{i \alpha} d_{2,1,0}(\beta)=-D_{2,1,0}^{*} \\
D_{2,-2,0}=D_{2,2,0}^{*} .
\end{array}
$$

\section{References}

[1] M. Rapini, R. A. Dias, and B. V. Costa, "Phase transition in ultrathin magnetic films with long-range interactions: Monte Carlo simulation of the anisotropic Heisenberg model," Phys. Rev. B, vol. 75, p. 014425, 2007.

[2] M. Cacciola and A. Berdie, "Studies on approximation methods in calculating the magnetic dipolar interaction energy, and its impact on the relaxation time of magnetic nanoparticle systems," Acta Phys. Pol. A, vol. 129, pp. 88-96, 2016.

[3] R. F. L. Evans, W. J. Fan, P. Chureemart, T. A. Ostler, M. O. A. Ellis, and R. W. Chantrell, "Atomistic spin model simulations of magnetic nanomaterials," J. Phys.: Condens. Matter, vol. 26, p. 103202, 2014.

[4] D. Serantes, V. Vega, W. O. Rosa, V. M. Prida, B. Hernando, M. Pereiro, and D. Baldomir, "Interplay between magnetic anisotropy and dipolar interaction in one-dimensional nanomagnets: Optimized magnetocaloric effect," Phys. Rev. B, vol. 86, p. 104431, 2012.

[5] D. Serantes, D. Baldomir, M. Pereiro, B. Hernando, V. M. Prida, J. L. S. Llamazares, A. Zhukov, M. Ilyn, and J. González, "Magnetocaloric effect in dipolar chains of magnetic nanoparticles with collinear anisotropy axes," Phys. Rev. B, vol. 80, p. 134421, 2009.

[6] D. Serantes, D. Baldomir, M. Pereiro, B. Hernando, V. M. Prida, J. L. S. Llamazares, A. Zhukov, M. Ilyn, and J. González, "Magnetic ordering in arrays of one-dimensional nanoparticle chains," J. Phys. $D$, vol. 42, p. 215003, 2009.

[7] E. Y. Vedmedenko, H. P. Oepen, and J. Kirschner, "Microstructure of the spin reorientation transition in second-order approximation of magnetic anisotropy," Phys. Rev. B, vol. 66, p. 214401, 2002

[8] P. P. Ewald, "Die Berechnung optischer und elektrostatischer Gitterpotentiale," Ann. Physik, vol. 64, pp. 253-287, 1921.

[9] S. W. de Leeuw, J. W. Perram, and E. R. Smith, "Simulation of electrostatic systems in periodic boundary conditions. I. Lattice sums and dielectric constants," Proc. R. Soc. A, vol. 373, pp. 27-56, 1980.

[10] H. G. Petersen, "Accuracy and efficiency of the particle mesh Ewald method," J. Chem. Phys., vol. 103, pp. 3668-3679, 1995.

[11] Z. Wang and C. Holm, "Estimate of the cutoff errors in the Ewald summation for dipolar systems," J. Chem. Phys., vol. 115, pp. 63516359, 2001.

[12] R. Courant and D. Hilbert, Methods of Mathematical Physics. Volume I. New York: Wiley-Interscience, 1962.

[13] C. D. H. Chisholm, Group Theoretical Techniques in Quantum Chemistry. New York: Academic Press, 1976.

[14] M. E. Rose, Elementary Theory of Angular Momentum. New York: Wiley, 1957.

[15] M. E. Rose, Relativistic Electron Theory. New York: Wiley, 1961. 